\title{
Local control of parameningeal rhabdomyosarcoma: An expert consensus guideline from the International Soft Tissue Sarcoma Consortium (INSTRuCT)
}

\author{
Dana Casey ${ }^{1}$, Henry Mandeville ${ }^{2}$, Julie Bradley ${ }^{3}$, Simone ter Horst ${ }^{4}$, Anthony Sheyn ${ }^{5}$, \\ Beate Timmermann ${ }^{6}$, and Suzanne Wolden ${ }^{7}$ \\ ${ }^{1}$ University of North Carolina at Chapel Hill School of Medicine \\ ${ }^{2}$ The Royal Marsden NHS Foundation Trust \\ ${ }^{3}$ U. Florida Proton Center \\ ${ }^{4}$ University Medical Center Utrecht, Princess Máxima Center for Pediatric Oncology \\ ${ }^{5}$ University of Tennessee Health Science Center \\ ${ }^{6}$ Westdeutsches Protonenthearpiezentrum \\ ${ }^{7}$ Memorial Sloan Kettering Cancer Center
}

March 4, 2022

\begin{abstract}
The International Soft-Tissue Sarcoma Database Consortium (INSTRuCT) consists of a collaboration between the Children's Oncology Group (COG) Soft Tissue Sarcoma Committee, the European pediatric Soft-Tissue Sarcoma Study Group (EpSSG), and the Cooperative Weichteilsarkom Studiengruppe (CWS). As part of the larger initiative of INSTRuCT to provide consensus expert opinions for clinical treatment of pediatric soft tissue sarcoma, we sought to provide updated, evidenced-based consensus guidelines for local treatment of parameningeal rhabdomyosarcoma using both existing literature as well as recommendations from the relevant cooperative group clinical trials. Overall, parameningeal rhabdomyosarcoma represents a distinctly challenging disease to treat given its location near many critical structures in the head and neck, frequently advanced local presentation, and predilection for local failure. Definitive chemoradiation remains the standard treatment approach for parameningeal rhabdomyosarcoma, with surgery often limited to biopsy or salvage therapy for recurrent disease. In this consensus paper, we specifically discuss consensus guidelines and evidence for definitive local management with radiotherapy, with a focus on imaging for radiotherapy planning, dose and timing of radiation, approach for nodal irradiation, various radiation techniques including proton therapy, and the limited role of surgical resection.
\end{abstract}

Local control of parameningeal rhabdomyosarcoma: An expert consensus guideline from the International Soft Tissue Sarcoma Consortium (INSTRuCT)

Dana L. Casey, $\mathrm{MD}^{1,2^{*}}$, Henry Mandeville, $\mathrm{MD}^{3}$, Julie A Bradley, $\mathrm{MD}^{4}$, Simone A.J. ter Horst, MD PhD ${ }^{5}$, Anthony Sheyn, $\mathrm{MD}^{6}$, Beate Timmermann, $\mathrm{MD}^{7}$, Suzanne L. Wolden, $\mathrm{MD}^{8}$

${ }^{1}$ Department of Radiation Oncology, University of North Carolina School of Medicine, Chapel Hill, NC

${ }^{2}$ Lineberger Comprehensive Cancer Center, University of North Carolina Hospitals, Chapel Hill, NC

${ }^{3}$ Royal Marsden NHS Foundation Trust, London, United Kingdom; Institute of Cancer Research, London, United Kingdom

${ }^{4}$ Department of Radiation Oncology, University of Florida, Jacksonville, FL 
${ }^{5}$ Department of Radiology, University Medical Center Utrecht, Princess Máxima Center for Pediatric Oncology, Utrecht, the Netherlands

${ }^{6}$ Department of Surgery, Division of Otolaryngology, University of Tennessee Health Science Center, Memphis, Tennessee

${ }^{7}$ Department of Particle Therapy, University Hospital Essen, West German Proton Therapy Centre Essen (WPE), West German Cancer Center (WTZ), German Cancer Consortium (DKTK), Essen, Germany

${ }^{8}$ Department of Radiation Oncology, Memorial Sloan Kettering Cancer Center, New York, NY

Brief Running title: Parameningeal rhabdomyosarcoma: INSTRuCT consensus

${ }^{*}$ Corresponding Author : Dana L. Casey, MD University of North Carolina School of Medicine, Department of Radiation Oncology, 101 Manning Drive CB \#7512; Tel: (984)974-0400; E-mail: dana_casey@med.unc.edu

Abstract word count: 185

Text word count: 5806

Tables: 1

Figures: 1

Keywords: rhabdomyosarcoma, radiation therapy, parameningeal, local control

Abbreviations

\begin{tabular}{ll}
\hline CBCT & Cone-beam computed tomography \\
\hline CTV & Clinical target volume \\
COG & Children's Oncology Group \\
CT & Computerized tomography \\
CTCAE & Common Toxicities Criteria on Adverse Events \\
CWS & Cooperative Weichteilsarkom Studiengruppe \\
DW & Diffusion weighted \\
EpSSG & European paediatric Soft tissue sarcoma Study Group \\
GTV & Gross tumor volume \\
IMPT & Intensity-modulated proton therapy \\
IMRT & Intensity-modulated radiotherapy \\
INSTRuCT & International Soft Tissue Sarcoma Database Consortium \\
IRSG & International rhabdomyosarcoma study group \\
MRI & Magnetic resonance imaging \\
OAR & Organs at risk \\
PENTEC & Pediatric Normal Tissue Effects in the Clinic \\
PET & Positron emission tomography \\
PM-RMS & Parameningeal rhabdomyosarcoma \\
PT & Proton therapy \\
PTV & Planning target volume \\
RT & Radiotherapy \\
SLN & Sentinel lymph node \\
\hline
\end{tabular}

\section{ABSTRACT}

The International Soft-Tissue Sarcoma Database Consortium (INSTRuCT) consists of a collaboration between the Children's Oncology Group (COG) Soft Tissue Sarcoma Committee, the European pediatric Soft-Tissue Sarcoma Study Group (EpSSG), and the Cooperative Weichteilsarkom Studiengruppe (CWS). 
As part of the larger initiative of INSTRuCT to provide consensus expert opinions for clinical treatment of pediatric soft tissue sarcoma, we sought to provide updated, evidenced-based consensus guidelines for local treatment of parameningeal rhabdomyosarcoma using both existing literature as well as recommendations from the relevant cooperative group clinical trials. Overall, parameningeal rhabdomyosarcoma represents a distinctly challenging disease to treat given its location near many critical structures in the head and neck, frequently advanced local presentation, and predilection for local failure. Definitive chemoradiation remains the standard treatment approach for parameningeal rhabdomyosarcoma, with surgery often limited to biopsy or salvage therapy for recurrent disease. In this consensus paper, we specifically discuss consensus guidelines and evidence for definitive local management with radiotherapy, with a focus on imaging for radiotherapy planning, dose and timing of radiation, approach for nodal irradiation, various radiation techniques including proton therapy, and the limited role of surgical resection.

\section{INTRODUCTION}

Rhabdomyosarcoma is the most common soft tissue sarcoma in children, with parameningeal tumors accounting for $15-20 \%$ of all cases. ${ }^{1}$ Parameningeal rhabdomyosarcoma (PM-RMS) specifically refers to those tumors arising from the nasopharynx, nasal cavity, paranasal sinuses, parapharyngeal space, pterygopalantine fossa, masticator space, mastoid cavity, and middle ear and are distinguished anatomically and prognostically from the more favorable tumors arising from the orbit and other head and neck sites. ${ }^{2}$ Approximately $50 \%$ of patients with PM-RMS will present with lymph node involvement and $25 \%$ with distant metastases. ${ }^{3}$ Intracranial extension, skull base erosion, and cranial nerve palsy all represent poor prognostic factors related to the local spread of disease. Embryonal histology is the most common pathologic subtype, representing approximately $70 \%$ of all parameningeal tumors. ${ }^{4,5}$

Overall, the prognosis for PM-RMS is unfavorable, with a 10 year event free survival of $<65 \%$ and overall survival $<70 \%$ from pooled historical data. ${ }^{4} \mathrm{PM}-\mathrm{RMS}$ remains a particularly challenging disease site to treat given its anatomical location and predilection for local and leptomeningeal failure. Local relapse results not only in severe loss of form and function in many patients, but also an unacceptably high rate of mortality, with a 3-year survival after local failure of $<20 \% .{ }^{6}$ Given the locally advanced presentation of PM-RMS and the anatomical constraints of the head and neck, gross total resection is usually not feasible due to the risks of severe cosmetic and functional morbidity. As such, definitive chemoradiation remains the preferred initial treatment modality for PM-RMS, with surgery most commonly limited to an initial biopsy to establish the diagnosis.

In this consensus review from the International Soft-Tissue Sarcoma Consortium [INSTRuCT, consisting of the Children's Oncology Group (COG), European Pediatric Soft-Tissue Sarcoma Group (EpSSG), and Cooperative Weichteilsarkom Studiengruppe (CWS)], we provide treatment recommendations for the local management of PM-RMS.

\section{CONSENSUS GUIDELINES}

\subsection{Imaging for radiotherapy planning}

\subsubsection{Expert Consensus}

Imaging in rhabdomyosarcoma plays a pivotal role for staging, response assessment, planning of local therapy (radiotherapy and/or surgery), relapse monitoring, and diagnosis of acute and late complications of treatment. To guide the optimal radiotherapy plan, it is essential to image the primary tumor and assess for metastatic sites. For the primary tumor, magnetic resonance imaging (MRI) is preferred over computerized tomography (CT) due to superior soft tissue contrast resolution and the absence of ionizing radiation, although CT is helpful for detection of bony involvement. PM-RMS is preferably imaged on a 3T MRI scanner because of its higher resolution in this radiologically challenging anatomical site, although imaging on a $1.5 \mathrm{~T}$ MRI scanner is an acceptable alternative.

For radiotherapy planning, the essential MRI sequences are the morphological sequences (coronal and axial T1-, T2-weighted sequences with fat suppressed T2 sequences) and those with the administration of 
intravenous contrast such as gadolinium (coronal and axial T1 post- contrast with and/or without fat suppression). In specific cases, extra sagittal sequences can aid delineation as can volumetric 3D T1 post contrast sequences which allow for reformatting of the tumor site from all different views. Diffusion Weighted (DW) MRI adds to tumor characterization and differentiation of active residual tumor from reactive surrounding edema, and DW-MRI allows evaluation of tumor response, which can also inform radiotherapy planning. Including DW imaging in the radiotherapy planning protocol represents a key opportunity to integrate response evaluation with optimization of imaging to create the most accurate radiotherapy plan. If feasible, combining and integrating MRI sequences for response assessment and radiotherapy planning can also save the younger patients an additional course of anesthesia for separate scans.

For the detection of locoregional lymph nodes and distant metastases, [F18]-2-fluoro-2-deoxygluose (FDG)positron emission tomography (PET) or FDG-MRI in combination with chest CT are the recommended investigations of choice, similar to non-PM-RMS. Histologic evaluation of any suspicious head and neck nodes seen on PET is recommended to confirm pathologic involvement.

\subsubsection{Evidence}

Pediatric deep or extensive soft tissue tumors, such as RMS arising from the head and neck region, are best depicted with MRI (preferably 3T) due to excellent spatial resolution, superior soft-tissue contrast, and multi-planar imaging. ${ }^{7,8}$ These properties allow for superior delineation of the extent of the tumor and involvement of adjacent structures compared to CT. This is especially critical in PM-RMS, where delineation of the extent of intracranial extension (with increased risk for focal or diffuse leptomeningeal spread), the different head and neck compartments, the boundaries with vascular and/or neural structures determining perineural spread, and invasion of bone, are of utmost importance. ${ }^{9,10}$ Additional CT imaging is helpful in some cases for detection of the extent of bone destruction and is usually acquired as part of the radiotherapy planning process. Raney et al. ${ }^{2,11}$ and Michalsky et al. ${ }^{6}$ showed that outcome in patients with PM-RMS significantly improved when high-risk features were clearly identified with MRI, highlighting the value of the MRI information in contributing to optimized treatment protocols and radiotherapy planning. DW series are standard of care in many centers given their ability to facilitate the differentiation between active residual disease and reactive surrounding tissue. However, there is still a need for systematic prospective evaluation of its role in diagnosis and response evaluation in PM-RMS, and this is being evaluated in the current EpSSG FaR RMS study. ${ }^{12,13}$

Regarding the role FDG-PET, a systemic review reported a sensitivity of $80-100 \%$ and $95-100 \%$ with a specificity of $89-100 \%$ and $80-100 \%$, respectively, for locoregional lymph nodes and non-pulmonary distant metastases. ${ }^{14,15}$ Pathologic confirmation of any suspicious locoregional nodes seen on FDG-PET is recommended at this time given the potential for false positives and subsequent impact on radiation planning and treatment.

2.2. Timing of radiation2.2.1 Expert Consensus For patients with locoregional PM-RMS, local therapy with definitive radiotherapy, or in very rare cases, delayed surgical excision of the primary tumor prior to radiotherapy, should commence at week 13 ; this approximates to the $5^{\text {th }}$ cycle of induction chemotherapy. Earlier radiotherapy (beginning at approximately week 4 to 6 ) may be considered for certain high risk scenarios, including the presence of intracranial extension where the risk of leptomeningeal relapse is higher and/or in the setting of reduced dose cyclophosphamide. In the rare case in which delayed surgical resection is performed, patients with locoregional PM-RMS requiring postoperative radiotherapy should commence radiation within 4- 6 weeks of surgery, usually with the $2^{\text {nd }}$ cycle of postoperative chemotherapy. For patients with metastatic PM-RMS, local therapy can be delivered at week 13 as is done in the patients with localized disease or can be delayed until week 20- 22 .

\section{Evidence}

The timing of local therapy, including radiotherapy, in the setting of multimodality treatment for these often highly chemotherapy sensitive tumors, has become increasingly aligned in recent international collaborative group protocols where previous significant differences existed. However, there are still some significant 
points of debate, and concerns have been raised that these recommended timings may impact local control for PM-RMS, particularly for those with high-risk features. ${ }^{16,17}$ In European studies, the approach used in both the EpSSG and CWS studies for patients with localized PM-RMS is to evaluate the response to induction chemotherapy after the $3^{\text {rd }}$ cycle, and then plan for the commencement of optimal local therapy at week 13 , approximating to the $5^{\text {th }}$ cycle of chemotherapy. Outcomes from the first RMS 2005 study randomization of EpSSG high risk rhabdomyosarcoma, where $33 \%$ of cases were parameningeal, reported the 3-year event free survivals to be $67.5 \%$ and $63.3 \%$ in the 2 arms, both significantly improved compared to historical cohorts, although the local failure outcomes are still to be reported. ${ }^{18}$ In the current EpSSG FaR-RMS study (NCT04625907), these timings continue to be recommended. By comparison, the approach for PM-RMS within COG has employed different timings, previously recommending the delivery of earlier radiotherapy given concerns about achieving local control for these challenging tumors. In these earlier protocols through the Intergroup Rhabdomyosarcoma Study Group (IRSG), there was a recommendation to commence radiotherapy at day 0 . A retrospective analysis of patients treated in the IRS-IV and D9803 studies revealed no significant difference in clinical outcomes in those receiving early radiotherapy compared to patients commencing radiotherapy at week 12 , with 5 -year local failure rates of $19 \%$ in both studies ${ }^{19}$ Similarly, there was no difference in local control based on timing of radiation (before 4 weeks or week 12-13) in a retrospective series from Memorial Sloan Kettering. ${ }^{20}$ The ARST0531 study, which was open from 2006 until 2012, recommended radiotherapy at week 4 with the goal of improving local control, yet the local control in this study was actually inferior to the previous study, D9803. ${ }^{17}$ However, there are some important confounding factors that make a direct comparison regarding timing difficult, with the dose of cyclophosphamide lower in ARST0531, and $47 \%$ of patients with PM-RMS on D9803 received radiotherapy at week 1 . The proximity of the parameningeal site to the skull base and cranial nerves, and the infiltrative pattern of growth observed with rhabdomyosarcoma means that radical delayed surgical resection of the primary tumor is very rarely undertaken as discussed in more detail below. Although not recommended, for those rare cases where delayed primary excision is done, postoperative radiotherapy is indicated. Adjuvant chemotherapy is usually commenced 2 weeks after surgery, with radiotherapy commencing as soon as possible after this once the surgical wounds have healed sufficiently (although delays may be necessary for postoperative complications). Preoperative radiotherapy has not yet been fully evaluated in rhabdomyosarcoma, and although it is being investigated in the FaR-RMS study, it is unlikely to be considered for PM-RMS given the complexities and potential complications of surgery. For metastatic PM-RMS, the recommended timings for radiotherapy have historically been at later time points (week 20-22) in the international collaborative group studies from both sides of the Atlantic. However, on the now open high-risk COG study, ARST2031, radiation to the primary site is delivered at week 12-13 for all tumors including PM-RMS (similar to patients with localized disease), with this timing representing an acceptable option for treatment of metastatic PM-RMS. European recommendations in the now closed BERNIE and MTS-2008 studies were for local therapy to commence at week 22, approximating to the $8^{\text {th }}$ cycle of chemotherapy, with re-evaluation of cases after the $6^{\text {th }}$ cycle of chemotherapy. Yet, it is apparent that these guidelines were not always adhered to, particularly with the challenges of treating metastatic rhabdomyosarcoma and multiple disease sites leading to delays in radiotherapy. In the analysis of the BERNIE study, a cut off of day 221 was used to define radical radiotherapy to take this issue into account. ${ }^{21}$ Cases with extensive metastatic disease may require metastatic radiotherapy delivered in sequential phases, treated in succession to limit the associated bone marrow and other acute toxicities. Delayed radiotherapy for metastatic sites is also recommended in COG studies, with metastatic site radiotherapy to now occur at approximately week 40 after completion of primary chemotherapy on ARST2031 (previously, on the prior high-risk study studies, ARST0431 and ARST08P1, there was the option to deliver metastatic site radiotherapy either with primary site radiation or after completion of primary chemotherapy).2.3 Definitive radiation dosing and volumes

\section{Expert Consensus}

As surgical resection is typically limited to a biopsy alone at diagnosis (without upfront gross total resection), the majority of patients with PM-RMS will have IRS group III disease. The definitive radiation dose for treatment of the primary site is 50.4 Gy in 28 fractions, similar to the dose delivered for group III tumors at 
other sites. A boost to 55.8 Gy or 59.4 Gy total is permitted for treatment of parameningeal tumors at the discretion of the treating physician, with unfavorable features such as tumor size [?] $5 \mathrm{~cm}$ at diagnosis, poor response to induction chemotherapy, or high-risk meningeal features such as intracranial extension. Volume reduction is also permitted to account for the response to initial chemotherapy. In these cases, the prechemotherapy extent of disease should be treated to 36 Gy - 41.4 Gy in 20-23 fractions, with a boost to the gross residual disease that is present at the time of radiation planning to the final total dose of 50.4 Gy - 59.4 Gy. The boost may be administered either sequentially as a cone-down or concurrently as a simultaneous integrated boost with feasible fraction sizes. The pre-chemotherapy and post-chemotherapy/pre-radiation imaging including the MRI should be fused to the CT simulation scan for optimal target delineation as discussed above. The gross tumor volume at diagnosis as defined by imaging and physical exam should be transferred to a tumor volume adapted to the actual geometry at time of radiotherapy planning (taking into account any anatomical shift due to tumor shrinkage or growth). This volume should be expanded by approximately $1 \mathrm{~cm}$, edited for anatomical barriers to spread, to account for occult tumor volume and create the pre-chemotherapy clinical target volume or CTV1 (also known as CTVp_pre). Careful attention should be given to including any initial extent of focal dural involvement in these volumes. The gross tumor volume at the time of radiation planning as defined by imaging and physical exam after several weeks of induction chemotherapy should also be expanded by approximately $0.5-1 \mathrm{~cm}$, edited for anatomical barriers to spread, to create the post-chemotherapy clinical target volume or CTV2 (also known as CTVp_post). An intermediate volume consisting of the prechemotherapy extent of tumor without a margin can also be utilized as is done in the CWS group. The planning target volume then typically consists of a geometric 3-5mm expansion on each clinical target volume to account for variability in daily set-up, although this expansion is uniquely defined for proton therapy. Of note, although radical surgery is not standard of care, if pursued, adjuvant radiation is often required due to fusion status, lack of ability to obtain negative margins, and/or regional nodal involvement. In PM-RMS, inclusion of the entire surgical scar or even surgical route is not often recommended as it may mean extensive radiotherapy volumes in some children. See Table 1 for dosing recommendations by clinical group and fusion status, including those with IRS group I-II disease.

\section{Evidence}

Over successive IRS protocols, radiation volumes for parameningeal tumors drastically decreased with the goal of maximizing the therapeutic ratio, from craniospinal irradiation in the late 1970s and whole brain radiation in the 1980s for those with high-risk meningeal features, to focal radiation for all patients in the $1990 \mathrm{~s}$, consisting of a 1-2cm margin around the gross tumor. ${ }^{2}$ Importantly, omission of whole brain radiation in lieu of focal radiation volumes did not compromise outcomes in an analysis of IRS II-IV ${ }^{2,6}$ including risk of local failure and risk of central nervous system recurrence.

Regarding the evolution of radiation dose for parameningeal tumors, on IRS-II and III, radiation dose depended on both the size of the tumor and age of the patient, with a median dose of approximately 45 Gy and 50.4 Gy delivered for those $<6$ years of age and $>6$ years of age, respectively. On IRS-IV, patients were randomized to receive either 50.4 Gy in 1.8 Gy fractions delivered daily or 59.4 Gy in 1.1 Gy fractions delivered twice daily, with similar local relapse rates observed in both arms. ${ }^{22}$ Overall, doses less than 47.5 Gy have been associated with worse local control for parameningeal tumors, ${ }^{6}$ in part supporting the radiation doses currently utilized on the ongoing protocols through the COG, EpSSG, and CWS. Previous smaller studies had also identified a similar relationship between improved local control and higher radiation doses for PM-RMS. ${ }^{23,24}$

Even with these doses, local relapse remains the dominant form of failure for PM-RMS, with localized failure seen in approximately $70 \%$ of those who relapse on both European and North American cooperative group trials. The majority of local failures occur in the high dose region, ${ }^{25-27}$ suggesting that marginal misses or volumetric coverage are not the issue. Furthermore, the utilization of a post-chemotherapy cone-down volume (to reduce the high-dose treatment volume) does not appear to compromise local control. ${ }^{28,29}$ Unfortunately, local failure for parameningeal tumors has actually worsened over time on the most recent COG studies from $16 \%$ on IRS-IV,$^{22}$ to $19 \%$ on D9803, ${ }^{30}$ to $28 \%$ on ARST $0531^{17}$ with similarly high rates of local failure seen 
in recent single institution studies, ${ }^{16,20,27}$ thought in part to be due to the lower doses of cyclophosphamide utilized on recent trials. In addition, leptomeningeal failure is a common and devastating pattern of relapse among patients with PM-RMS, especially among those with intracranial extension at diagnosis. ${ }^{31-33}$

Ongoing strategies to overcome the possibly radioresistant phenotype of parameningeal tumors include radiation dose escalation. Hyperfractionation to 59.4 Gy on IRS-IV did not result in increased local control compared to 50.4 Gy with daily fractionation, although 59.4 Gy delivered in 54 fractions twice a day may not deliver a more biologically effective dose than 50.4 Gy in 28 daily fractions. Thus, current efforts to mitigate the worsening local control include radiation dose escalation to 59.4 Gy with daily fractionation in tumors $>$ $5 \mathrm{~cm}$, as larger tumor size has consistently been associated with increased local failure rates. ${ }^{4,17,30,34}$ Given the infiltrative nature, location near many critical structures, and utilization of concurrent chemotherapy, escalation beyond 59.4 Gy may not be feasible, and doses $>57$ Gy did not show a benefit on the CWS studies. ${ }^{35}$ In the FaR-RMS study, all patients with PM-RMS are eligible to be randomized between 50.4 Gy and 59.4 Gy, which will provide future guidance regarding the true benefit of dose escalation for these tumors. Improvements in systemic therapy such as with higher doses of alkylating agents and/or introduction of concurrent radiosensitizers are promising for reducing the incidence of local failure. In addition, given the key role of radiotherapy in the definitive treatment of PM-RMS, efforts to identify molecular determinants of radiation response are critical to enhance local control and outcomes for patients with parameningeal disease.

\subsection{Nodal irradiation}

\subsubsection{Expert Consensus:}

For node negative PM-RMS, prophylactic nodal irradiation is not recommended. For node positive PM-RMS (clinical or pathologic involvement), 36-41.4 Gy at 1.8 Gy per fraction is recommended to the involved nodal chain or site. For nodal volumes, the GTV consists of the gross nodal disease, with a $1 \mathrm{~cm}$ expansion edited for normal tissue barriers to disease extension to create the CTV. The CTV should also encompass the entire lymph node drainage chain or, as per EpSSG practice, a 2-3 cm expansion superiorly and inferiorly in the direction of nodal drainage, using a involved site approach as an acceptable alternative. The CTV is then expanded by $3-5 \mathrm{~mm}$ for PTV. The recommended dose to grossly involved nodes at the time of radiation planning is 50.4 Gy at 1.8 Gy per fraction, while 36-41.4 Gy is sufficient for nodes that have had a complete response to chemotherapy at the time of radiation.

\subsubsection{Evidence:}

Nodal involvement at diagnosis and regional recurrence after definitive therapy are known patterns of spread in PM-RMS. In a series of 94 patients with RMS (no elective nodal irradiation), $21 \%$ developed regional recurrence and two-thirds of these relapses occurred in patients with a head and neck primary site. ${ }^{36} \mathrm{~A}$ larger percent of patients developed nodal metastases in a smaller series of head and neck RMS that included only patients with alveolar histology. ${ }^{37}$ After a median follow-up of 4.3 years, with regional radiation targeted only to involved nodes, $75 \%$ of patients with N0 disease developed isolated nodal relapse in the ipsilateral first-echelon nodal basin, and 33\% of patients with N1 disease developed nodal relapse, one isolated and one concomitantly with local progression.

Given the concern for nodal progression particularly in alveolar RMS, prophylactic nodal irradiation has been investigated. In a retrospective series consisting of 47 patients with PM-RMS treated with definitive IMRT to 50.4 Gy in 28 fractions and chemotherapy, patients [?] 14 years of age with alveolar histology ( $\mathrm{n}=13)$ were treated to 36 Gy at 1.3 Gy per fraction the bilateral cervical nodal chains. ${ }^{33}$ Ten of these patients had N1 disease, and gross nodal disease was boosted to 50.4 Gy. Of patients with embryonal histology, none experienced regional failure (though the number with N1 disease was not reported), nor did any with alveolar histology who received bilateral cervical nodal radiation. Of those with alveolar histology who did not receive bilateral cervical nodal radiation, the 5 year regional failure rate was $37 \%$. However, distant disease (including CNS dissemination) remained the dominant pattern of failure in these patients. Another series of 46 patients with head and neck rhabdomyosarcoma yielded similar results. In this cohort, 5-year 
regional control rate was $73 \% .{ }^{31}$ Those with alveolar histology experienced higher rates of nodal recurrence or progression compared to embryonal histology, with 5 year regional control rate of $37 \%$ vs $88 \%$, respectively.

However, other studies have found lower rates of regional recurrence with irradiation of only the involved nodal basin for patients with node positive disease, without prophylactic nodal irradiation. In a retrospective review of a prospective database, patterns of failure were reported on 66 children with RMS across diverse primary sites. ${ }^{38}$ Overall, 7 patients developed local recurrence ( $\mathrm{n}=6$ with embryonal histology), achieving $88 \%$ 2-year local control for the cohort. No patients with parameningeal disease developed regional failure. Another series that used proton therapy for the treatment of RMS across primary sites reported no regional failures, with radiotherapy directed only to involved nodal levels. ${ }^{25}$ In a retrospective review of 24 patients with alveolar PM-RMS in which radiation was directed only to the involved nodal level(s) with a boost to the involved nodes, 3 year regional control was $94 \% .{ }^{32}$ One patient with a node negative nasal cavity primary developed a submental nodal recurrence. None of the node-positive patients treated with nodal irradiation developed regional progression, though node positive disease was associated with inferior overall survival due to distant disease progression. An interim report on RMS13 described concerning rates of disease progression in patients with PM-RMS receiving reduced dose cyclophosphamide, prompting a change in the timing of radiotherapy. ${ }^{16}$ Local and CNS progression predominated, with no patients developing nodal relapse.

Gallego et al. reported on a combined analysis of patients with node positive alveolar RMS treated on EpSSG RMS 2005 and COG ARST0531, with parameningeal primaries in 34\% and 25\%, respectively. ${ }^{39}$ Metastatic progression comprised the dominant pattern of relapse in both trials. Nodal recurrence alone occurred in 12\% of patients in EpSSG RMS 2005 and 9\% on ARST0531, while nodal recurrence with local and/or metastatic progression occurred in an additional $10 \%$ and $8 \%$, respectively. Of note, of those who had regional nodes evaluated pathologically, positive nodes were identified in 92\% on EpSSG RMS 2005 and $74 \%$ in ARST0531, highlighting a discrepancy between clinically positive and pathologically positive nodes in rhabdomyosarcoma. Sentinel lymph node (SLN) biopsy has been evaluated as a possible tool for accurate nodal staging in patients with head and neck rhabdomyosarcoma. In a small series of 6 patients, 2 of 3 patients with radiographically suspicious nodes by PET and/or MRI had a positive SLN, while 1 of 3 with negative imaging had a positive SLN. ${ }^{40}$ Mapping of SLN yielded an unanticipated location of the SLN in 2 patients, with the nodes mapping in the contralateral neck. The use of SLN resulted in a change in the recommended treatment in 2 of the 6 patients. Overall, the use of SLN in PM-RMS remains investigational at the present time.

\section{Radiotherapy techniques}

\subsubsection{Expert Consensus}

Due to the proximity to relevant structures like the optic chiasm, brainstem, salivary and lacrimal glands, and pituitary, PM-RMS is a particularly challenging site when tailoring local therapy. Typical radiation doses, especially when combined with intensive chemotherapy, may exceed organ tolerance of the head and neck structures, particularly with regard to the young patient age. In order to maximize form and function and minimize toxicity, RT should be done with careful planning and attention to organs at risk (OAR), and dose constraints should be applied as per the Pediatric Normal Tissue Effects in the Clinic (PENTEC) report $^{41}$ and according to current study guidelines in these young, curable patients. In addition, functional assessments (ex. hearing, vision, neuroendocrine) are encouraged prior to starting radiation to serve as a baseline.

Several highly conformal radiation techniques are available that enable protection of healthy tissue while maximizing tumor control. For PM-RMS, predominantly techniques such as proton therapy (PT) or intensitymodulated RT (IMRT) should be used. In addition, immobilization with a face mask and careful image guidance with cone-beam computed tomography (CBCT) to ensure reliable daily set up are recommended. As many of the patients with PM-RMS may be too young to consciously cooperate, sedation with anesthesia may be required to safely perform daily radiotherapy.

For younger patients and for treatment of tumors located near critical structures such as those in the 
head and neck or near the brainstem, modern treatment concepts encourage the use of highly conformal techniques such as PT to minimize dose to normal tissue secondary to the steep dose gradient of PT. In general, intensity modulation in PT (IMPT) can offer additional benefits when compared to single field optimization PT techniques, especially if lymph nodes of the neck are involved. However, the use of PT is restricted to radiation oncologists experienced in this field with access to a proton center, and randomized studies comparing PT versus IMRT are scarce, with ethical concerns often hampering the conduction of such studies. Given the limitations in access to proton therapy, IMRT can be used in order to achieve highly conformal treatment as well. However, due to the high integral dose and low dose bath associated with IMRT, it is typically applied in older patients or whenever PT is not available or technically restricted (e.g. lack of gantries, lack of high energies). Additionally, as social, logistical, and financial issues may make PT prohibitive, IMRT may be preferred in those scenarios. In specialized programs, brachytherapy can also be explored, particularly in the locally recurrent setting or as part of clinical protocols like AMORE, a local treatment regimen consisting of Ablative surgery, Moulage technique brachytherapy and surgical Reconstruction. ${ }^{42-44}$

\subsubsection{Evidence}

Evolving techniques have improved the application of radiotherapy in the last decades for PM-RMS. IMRT has enabled better target dose coverage than three-dimensional conformal radiotherapy, ${ }^{45}$ and PT has further promised the reduction of unnecessary dose to critical structures in the head and neck such as the optic apparatus, hypothalamic-pituitary axis, brainstem, musculoskeletal structures, as well as salivary glands. Thus far, several studies have examined the application of PT for PM-RMS, resulting in similar oncologic outcomes as photon therapy, with overall survival rates ranging between $58 \%$ and $88.9 \%$ and local control rates between $66 \%$ and $84 \% .{ }^{25,26,32,46}$ Unfortunately, local failure in the high-dose region remains problematic after both IMRT and PT. ${ }^{20,25}$

Although local control is deemed the main focus of radiotherapy for pediatric rhabdomyosarcoma, late effects such as endocrine dysfunction, secondary cancers, and cosmetic outcomes like facial hypoplasia are a significant relevant issue, with the latter affecting up to $97 \%$ of treated patients. ${ }^{26,43,46}$ For PM-RMS specifically, dosimetric comparisons have shown that PT, as compared to IMRT, achieves improved sparing of relevant structures for a variety of organs like brainstem, pituitary, and ipsilateral and contralateral lacrimal and parotid glands. ${ }^{47}$ Regarding acute toxicity, a study examining PT versus IMRT for PM-RMS reported no overall difference in acute toxicity grade $<3$. However, the risk of experiencing grade 3 dermatitis was higher after PT, while the risk of experiencing grade 3 mucositis was higher after IMRT. ${ }^{20}$ Regarding long-term toxicity after IMRT for RMS of the head and neck, among the Memorial Sloan Kettering series, after a median follow up of 7.7 years, the most common long-term toxicity was facial disfigurement (observed in $77 \%$ of patients), with other common toxicities including growth hormone deficiency, dental problems, and cataracts (all seen in 30-40\% of patients). ${ }^{48}$ Overall, clinical reports on toxicity for PM-RMS have shown a low probability of adverse events after PT, although the number of patients in available studies and length of follow-up are limited. In an analysis of 46 children treated with PT, the mean maximal acute and late toxicity score according to Common Toxicities Criteria on Adverse Events (CTCAE) were 2.1 and 1.1, respectively and no acute or late toxicity exceeding grade 3 was reported. ${ }^{25}$ Importantly, within this study, the risk for a field-border or marginal recurrence was low. This is in accordance with other studies reporting low rates of acute or late toxicity after PT with a toxicity [?] grade 3 free survival of $95 \%$ at five years. ${ }^{26}$ A study from Massachusetts General Hospital looking at late toxicity after PT for rhabdomyosarcoma showed overall low rates of low toxicity (35\% late toxicity of any grade with median follow up of 47 months), with specific late toxicities in those with head and neck disease including facial hypoplasia (9\%), dry eye (9\%) and cataracts $(3 \%) .{ }^{49}$ With regard to second cancer risk, PT has also shown reduced risk when compared to IMRT across different cancer types, although prospective long-term comparison of the second cancer risk after PT versus IMRT is lacking. ${ }^{50}$

Brachytherapy only plays a minor role in current treatment of PM-RMS. As such, evidence for brachytherapy is limited. However, brachytherapy was used as part of upfront and salvage treatment after prior external 
beam RT in the AMORE protocol, and results seem to be promising. ${ }^{44}$

\subsection{Surgical resection}

\subsubsection{Expert Consensus}

Pediatric parameningeal tumors typically present with locally advanced disease secondary to the non-specific nature of the symptoms that they cause. Due to the anatomical constraints and infiltrative nature of parameningeal tumors, it is very difficult to obtain oncologically negative margins without compromise of form and function (for example, cranial nerve deficits, disfigurement of the facial bones). Surgical resection thus holds a minimal role in the upfront management of PM-RMS and should, for the most part, only be considered for non-parameningeal head and neck sites where resection can be done with minimal morbidity and little cosmetic/functional deficits. For PM-RMS specifically, surgical approaches have typically been limited to biopsy and salvage for recurrent disease. If resection is performed, a multidisciplinary team at a specialized center should be involved, including otolaryngology, neurosurgery, plastic surgery, and oral maxillofacial surgery.

\subsubsection{Evidence}

The large majority of patients with parameningal tumors ( $\sim 95 \%)$ will have IRS group III disease secondary to the anatomical difficulties and morbidity of performing a gross total resection at diagnosis. ${ }^{6,35}$ Surgery for parameningeal sites has been considered to be unfavorable, due to risk of disfigurement, injury to neurovascular structures, and difficulty in achieving complete resection. ${ }^{51,52}$ Common toxicities of surgical resection for parameningeal tumors can include cranial nerve palsy, CSF leaks, trismus, and poor cosmesis. ${ }^{1}$

With advances in endoscopic approaches and the quality of imaging, certain tumors within the nasopharynx, sinonasal cavities, and skull base without dural involvement may be amenable to endoscopic approaches at specialized treatment centers. ${ }^{53}$ If surgical resection is considered, smaller tumors $(<5 \mathrm{~cm}$, those without dural involvement) appear to be more amenable to resection relative to larger ones. ${ }^{51}$ Primary resection in smaller tumors may have advantages such as avoidance of short and long term morbidities associated with radiation to the pediatric skull base and sinonasal cavities as described in small series. ${ }^{51,53}$ However, there is often difficulty in obtaining negative margins, necessitating postoperative radiation therapy. ${ }^{52,54}$ The advancement of microsurgical techniques has also rendered surgery a treatment option in select cases. When amenable to resection, these tumors are usually approached through a transfacial or cranio-orbito-zygomatic approach, which requires reconstruction after tumor resection is completed. Use of free tissue transfer has enabled the ability to reconstruct areas once thought to be unreachable by pedicled vascular flaps. ${ }^{55}$

In the hands of experienced surgical teams, there appears to be a low risk of severe postoperative morbidity, although this is very dependent on experience. The morbidity of surgical resection combined with the potential need for adjuvant radiation must be carefully evaluated for each patient. ${ }^{53,56}$ In the minority of patients who were able to undergo initial resection with microscopic residual disease on COG protocols, the addition of surgery with resulting lower IRS group was not associated with an improvement in outcomes, ${ }^{4}$ and radiation continued to be delivered in the setting of microscopic residual disease and/or positive margins (IRS group II). In addition, when performed as a delayed primary excision or second look surgery, although radiation doses can be reduced to 36-41.4 Gy, radiation is still necessary, and there is no clear oncologic benefit from delayed primary excision in this setting for PM-RMS. ${ }^{57}$ In general, the tradeoff in morbidity between the reduction in radiation dose with the addition of surgery in the setting of delayed primary excision must be further explored with quality of life and late effect assessments on future rhabdomyosarcoma protocols. ${ }^{58}$ Overall though, without an oncologic benefit with the addition of surgery to radiation, the acute and long-term toxicity of trimodality therapy must be carefully considered and selectively applied.

\section{GUIDELINE SUMMARY (Fig. 1)}

PM-RMS represents a very challenging site to treat with local failure remaining the dominant form of failure. Definitive chemoradiation is the preferred treatment approach for PM-RMS, with the goal of maximizing 
cure while minimizing loss of form and function. Radiation timing, volumes, and techniques have evolved greatly over the last 50 years with this goal in mind.

Imaging with MRI of the head neck is critical in PM-RMS to assess the extent of disease (including any possible intracranial extension), evaluate response, and fuse for accurate radiation planning. Although radiotherapy was previously delivered earlier for those with high-risk parameningeal features, the timing of primary site radiotherapy for localized PM-RMS is now similar to other sites at week 13 (and up to week 20-22 in the metastatic setting). The recommended radiation dose for PM-RMS is 50.4 Gy in 28 fractions, with a boost to 55.8 - 59.4 Gy allowed for unfavorable features. To account for the response to chemotherapy prior to radiation, the pre-chemotherapy extent of disease can be treated to 36 Gy - 41.4 Gy, with a boost to the macroscopic residual disease present at the time of radiation planning to the final dose. Radiation target volumes have reduced drastically over time, with the goal of enhancing the therapeutic ratio, from prior craniospinal irradiation to now conformal treatment of the tumor with a $1-1.5 \mathrm{~cm}$ margin. Although prophylactic nodal irradiation is not recommended, for patients with clinical or pathologic involvement of nodes, a dose of 36-41.4 Gy over 20-23 fractions (with gross disease to $50.4 \mathrm{~Gy}$ ) is recommended to the involved nodal chain or site. To minimize toxicity and dose to the many critical organs at risk in the head and neck, highly conformal techniques like IMRT or proton therapy must be utilized, with proton therapy specifically encouraged whenever feasible to minimize normal tissue toxicity. Despite advancements in volumes and technology, morbidity of local therapy with radiation to the head and neck remains significant, including cosmetic defects, endocrinopathies, impaired vision and hearing, dental complications, and second cancers.

Given the locally infiltrative nature and difficulty with obtaining negative margins, the role of surgical resection is often limited to initial biopsy and for local therapy in the relapsed setting. However, constant reevaluation of the optimal treatment approach for patients with PM-RMS is necessary. Overall, we recommend a multi-disciplinary approach for treatment of PM-RMS, with the primary goal of maximizing cure while maintaining form and function.

\section{ACKNOWLEDGMENTS}

We would like to thank Suzi Birz for her organizational and administrative expertise. The International Soft Tissue Sarcoma Consortium and the Pediatric Cancer Data Commons are supported in part by Cancer Research Foundation, Children's Research Foundation, Comer Development Board, Kick Cancer, King Baudouin Foundation, Rally Foundation for Childhood Cancer Research, Seattle Children's Foundation from Kat's Crew Guild through the Sarcoma Research Fund, St. Baldrick's Foundation, and The Andrew McDonough B+ Foundation. This work is made possible through the efforts of Children's Oncology Group, Cooperative Weichteilsarkom Studiengruppe der GPOH, The European paediatric Soft tissue sarcoma Study Group, MMT Malignant Mesenchymal Tumour Committee, and STSC AIEOP Italian Soft Tissue Sarcoma Committee.

\section{CONFLICT OF INTEREST STATEMENT:}

DC, SH, AS, BT, SW have no relevant conflicts to disclose. HM acknowledges the support of the NIHR Biomedical Research Centre at The Royal Marsden NHS Foundation Trust and The Institute of Cancer Research. JB acknowledges a research grant from Asco/Pfizer.

\section{REFERENCES}

1 Casey, D. L. \& Wolden, S. L. Rhabdomyosarcoma of the Head and Neck: A Multimodal Approach. $J$ Neurol Surg B Skull Base 79, 58-64 (2018).

2 Raney, R. B., Jr. et al. Improved prognosis with intensive treatment of children with cranial soft tissue sarcomas arising in nonorbital parameningeal sites. A report from the Intergroup Rhabdomyosarcoma Study. Cancer 59, 147-155 (1987).

3 Turner, J. H. \& Richmon, J. D. Head and neck rhabdomyosarcoma: a critical analysis of population-based 
incidence and survival data.Otolaryngol Head Neck Surg 145, 967-973 (2011).

4 Merks, J. H. et al. Parameningeal rhabdomyosarcoma in pediatric age: results of a pooled analysis from North American and European cooperative groups. Ann Oncol 25 , 231-236 (2014).

5 Rahman, H. A. et al. Outcome of pediatric parameningeal rhabdomyosarcoma. The Children Cancer Hospital, Egypt, experience.J Egypt Natl Canc Inst 25 , 79-86 (2013).

6 Michalski, J. M. et al. Influence of radiation therapy parameters on outcome in children treated with radiation therapy for localized parameningeal rhabdomyosarcoma in Intergroup Rhabdomyosarcoma Study Group trials II through IV. Int J Radiat Oncol Biol Phys59 , 1027-1038 (2004).

7 Siegel, M. J. Magnetic resonance imaging of musculoskeletal soft tissue masses. Radiol Clin North Am 39 , 701-720 (2001).

8 Jawad, N. \& McHugh, K. The clinical and radiologic features of paediatric rhabdomyosarcoma. Pediatr Radiol 49 , 1516-1523 (2019).

9 Freling, N. J. et al. Imaging findings in craniofacial childhood rhabdomyosarcoma. Pediatr Radiol 40 , 1723-1738; quiz 1855 (2010).

10 Van Rijn, R. R. et al. Imaging findings in noncraniofacial childhood rhabdomyosarcoma. Pediatr Radiol 38, 617-634 (2008).

11 Raney, R. B. et al. Treatment of children and adolescents with localized parameningeal sarcoma: experience of the Intergroup Rhabdomyosarcoma Study Group protocols IRS-II through -IV, 1978-1997.Med Pediatr Oncol 38 , 22-32 (2002).

12 Norman, G. et al. Mind the gap: extent of use of diffusion-weighted MRI in children with rhabdomyosarcoma. Pediatr Radiol 45, 778-781 (2015).

13 Inarejos Clemente, E. J. et al. MRI of Rhabdomyosarcoma and Other Soft-Tissue Sarcomas in Children. Radiographics 40, 791-814 (2020).

14 Harrison, D. J., Parisi, M. T. \& Shulkin, B. L. The Role of (18)F-FDG-PET/CT in Pediatric Sarcoma. Semin Nucl Med47, 229-241 (2017).

15 Vaarwerk, B. et al. Fluorine-18-fluorodeoxyglucose (FDG) positron emission tomography (PET) computed tomography (CT) for the detection of bone, lung, and lymph node metastases in rhabdomyosarcoma. Cochrane Database Syst Rev 11, CD012325 (2021).

16 Lucas, J. T., Jr., Pappo, A. S., Wu, J., Indelicato, D. J. \& Krasin, M. J. Excessive Treatment Failures in Patients With Parameningeal Rhabdomyosarcoma With Reduced-dose Cyclophosphamide and Delayed Radiotherapy. J Pediatr Hematol Oncol 40 , 387-390 (2018).

17 Casey, D. L. et al. Increased local failure for patients with intermediate-risk rhabdomyosarcoma on ARST0531: A report from the Children's Oncology Group. Cancer 125 , 3242-3248 (2019).

18 Bisogno, G. et al. Addition of dose-intensified doxorubicin to standard chemotherapy for rhabdomyosarcoma (EpSSG RMS 2005): a multicentre, open-label, randomised controlled, phase 3 trial.Lancet Oncol 19 , 1061-1071 (2018).

19 Spalding, A. C. et al. The effect of radiation timing on patients with high-risk features of parameningeal rhabdomyosarcoma: an analysis of IRS-IV and D9803. Int J Radiat Oncol Biol Phys87, 512-516 (2013).

20 Casey, D. L., Wexler, L. H. \& Wolden, S. L. Worse Outcomes for Head and Neck Rhabdomyosarcoma Secondary to Reduced-Dose Cyclophosphamide.Int J Radiat Oncol Biol Phys 103 , 1151-1157 (2019).

21 Cameron, A. L. et al. The Impact of Radiation Therapy in Children and Adolescents With Metastatic Rhabdomyosarcoma. Int J Radiat Oncol Biol Phys 111, 968-978 (2021). 
22 Donaldson, S. S. et al. Results from the IRS-IV randomized trial of hyperfractionated radiotherapy in children with rhabdomyosarcoma-a report from the IRSG. Int J Radiat Oncol Biol Phys 51, 718-728 (2001).

23 Berry, M. P. \& Jenkin, R. D. Parameningeal rhabdomyosarcoma in the young. Cancer 48, 281-288 (1981).

24 Donaldson, S. S., Castro, J. R., Wilbur, J. R. \& Jesse, R. H., Jr. Rhabdomyosarcoma of head and neck in children. Combination treatment by surgery, irradiation, and chemotherapy. Cancer 31, 26-35 (1973).

25 Doyen, J. et al. Outcome and Patterns of Relapse in Childhood Parameningeal Rhabdomyosarcoma Treated With Proton Beam Therapy.Int J Radiat Oncol Biol Phys 105 , 1043-1054 (2019).

26 Weber, D. C. et al. Pencil Beam Scanning Proton Therapy for Pediatric Parameningeal Rhabdomyosarcomas: Clinical Outcome of Patients Treated at the Paul Scherrer Institute. Pediatr Blood Cancer63 , 1731-1736 (2016).

27 Ladra, M. M. et al. Local failure in parameningeal rhabdomyosarcoma correlates with poor response to induction chemotherapy. Int J Radiat Oncol Biol Phys 92, 358-367 (2015).

28 Chen, C., Shu, H. K., Goldwein, J. W., Womer, R. B. \& Maity, A. Volumetric considerations in radiotherapy for pediatric parameningeal rhabdomyosarcomas. Int J Radiat Oncol Biol Phys 55 , 1294-1299 (2003).

29 Eaton, B. R. et al. Radiation therapy target volume reduction in pediatric rhabdomyosarcoma: implications for patterns of disease recurrence and overall survival. Cancer 119 , 1578-1585 (2013).

30 Wolden, S. L. et al. Local Control for Intermediate-Risk Rhabdomyosarcoma: Results From D9803 According to Histology, Group, Site, and Size: A Report From the Children's Oncology Group. Int J Radiat Oncol Biol Phys 93, 1071-1076 (2015).

31 Ludmir, E. B. et al. Patterns of failure following proton beam therapy for head and neck rhabdomyosarcoma. Radiother Oncol134, 143-150 (2019).

32 Bradley, J. A. et al. Patterns of Failure in Parameningeal Alveolar Rhabdomyosarcoma. Int J Radiat Oncol Biol Phys107, 325-333 (2020).

33 Yang, J. C., Wexler, L. H., Meyers, P. A. \& Wolden, S. L. Parameningeal rhabdomyosarcoma: outcomes and opportunities. Int J Radiat Oncol Biol Phys 85, e61-66 (2013).

34 Benk, V. et al. Parameningeal rhabdomyosarcoma: results of an international workshop. Int $J$ Radiat Oncol Biol Phys 36 , 533-540 (1996).

35 Koscielniak, E. et al. Prognosis of patients with parameningeal rhabdomyosarcoma (PM-RMS): The long term results of five consecutive CWS studies 1981-2009. Journal of Clinical Oncology34 , 10529-10529 (2016).

36 Buszek, S. M. et al. Disease Control and Patterns of Failure After Proton Beam Therapy for Rhabdomyosarcoma. Int J Radiat Oncol Biol Phys 109 , 718-725 (2021).

37 Ludmir, E. B. et al. Regional Nodal Control for Head and Neck Alveolar Rhabdomyosarcoma. Int J Radiat Oncol Biol Phys101 , 169-176 (2018).

38 Vern-Gross, T. Z., Indelicato, D. J., Bradley, J. A. \& Rotondo, R. L. Patterns of Failure in Pediatric Rhabdomyosarcoma After Proton Therapy. Int J Radiat Oncol Biol Phys 96 , 1070-1077 (2016).

39 Gallego, S. et al. Alveolar rhabdomyosarcoma with regional nodal involvement: Results of a combined analysis from two cooperative groups. Pediatr Blood Cancer 68 , e28832 (2021).

40 Turpin, B. et al. Sentinel lymph node biopsy in head and neck rhabdomyosarcoma. Pediatr Blood Cancer 66 , e27532 (2019). 
41 Constine, L. S. et al. Pediatric Normal Tissue Effects in the Clinic (PENTEC): An International Collaboration to Analyse Normal Tissue Radiation Dose-Volume Response Relationships for Paediatric Cancer Patients. Clin Oncol (R Coll Radiol) 31, 199-207 (2019).

42 Buwalda, J. et al. A novel local treatment strategy for advanced stage head and neck rhabdomyosarcomas in children: results of the AMORE protocol. Eur J Cancer 39 , 1594-1602 (2003).

43 Schoot, R. A. et al. Adverse events of local treatment in long-term head and neck rhabdomyosarcoma survivors after external beam radiotherapy or AMORE treatment. Eur J Cancer 51, 1424-1434 (2015).

44 Vaarwerk, B. et al. AMORE treatment as salvage treatment in children and young adults with relapsed head-neck rhabdomyosarcoma.Radiother Oncol 131 , 21-26 (2019).

45 Lin, C. et al. Effect of radiotherapy techniques (IMRT vs. 3D-CRT) on outcome in patients with intermediate-risk rhabdomyosarcoma enrolled in COG D9803-a report from the Children's Oncology Group.Int J Radiat Oncol Biol Phys 82, 1764-1770 (2012).

46 Childs, S. K. et al. Proton radiotherapy for parameningeal rhabdomyosarcoma: clinical outcomes and late effects. Int J Radiat Oncol Biol Phys 82, 635-642 (2012).

47 Kozak, K. R., Adams, J., Krejcarek, S. J., Tarbell, N. J. \& Yock, T. I. A dosimetric comparison of proton and intensity-modulated photon radiotherapy for pediatric parameningeal rhabdomyosarcomas. Int J Radiat Oncol Biol Phys 74, 179-186 (2009).

48 Lockney, N. A. et al. Late Toxicities of Intensity-Modulated Radiation Therapy for Head and Neck Rhabdomyosarcoma. Pediatr Blood Cancer 63, 1608-1614 (2016).

49 Ladra, M. M. et al. Preliminary results of a phase II trial of proton radiotherapy for pediatric rhabdomyosarcoma. J Clin Oncol32, 3762-3770 (2014).

50 Xiang, M., Chang, D. T. \& Pollom, E. L. Second cancer risk after primary cancer treatment with threedimensional conformal, intensity-modulated, or proton beam radiation therapy. Cancer $\mathbf{1 2 6}, 3560-3568$ (2020).

51 Daya, H., Chan, H. S., Sirkin, W. \& Forte, V. Pediatric rhabdomyosarcoma of the head and neck: is there a place for surgical management? Arch Otolaryngol Head Neck Surg 126 , 468-472 (2000).

52 Zevallos, J. P. et al. Modern multimodality therapy for pediatric nonorbital parameningeal sarcomas. Head Neck32, 1501-1505 (2010).

53 Wertz, A. et al. Minimally Invasive Approach for Resection of Parameningeal Rhabdomyosarcoma. $J$ Neurol Surg B Skull Base78 , 210-214 (2017).

54 Callender, T. A. et al. Rhabdomyosarcoma of the nose and paranasal sinuses in adults and children. Otolaryngol Head Neck Surg 112, 252-257 (1995).

55 Carty, M. J., Ferraro, N. \& Upton, J. Reconstruction of pediatric cranial base defects: a review of a single microsurgeon's 30-year experience. J Craniofac Surg 20 Suppl 1, 639-645 (2009).

56 Reilly, B. K. et al. Rhabdomyosarcoma of the head and neck in children: review and update. Int $J$ Pediatr Otorhinolaryngol79 , 1477-1483 (2015).

57 Rodeberg, D. A. et al. Delayed primary excision with subsequent modification of radiotherapy dose for intermediate-risk rhabdomyosarcoma: a report from the Children's Oncology Group Soft Tissue Sarcoma Committee. Int J Cancer 137, 204-211 (2015).

58 Lautz, T. B. et al. Benefit of delayed primary excision in rhabdomyosarcoma: A report from the Children's Oncology Group.Cancer 127 , 275-283 (2021). 
FIGURE 1 INSTRuCT consensus guideline summary for local management of parameningeal rhabdomyosarcoma

\section{Hosted file}

table 1 pm-rms pbc.docx available at https://authorea.com/users/452579/articles/558643local-control-of-parameningeal-rhabdomyosarcoma-an-expert-consensus-guideline-from-theinternational-soft-tissue-sarcoma-consortium-instruct

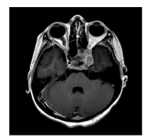

Imaging -MRI preferred T1, with and without contrast)

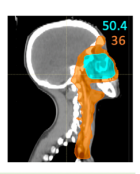

Dose/Volume

-Initial extent of disease and involved
nodes:
66-41.4 Gy Residual macrosco disease: $50.4-59.4 \mathrm{~Gy}$

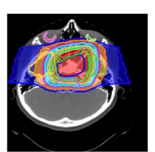

RT Technique

-Proton therapy

preferred

therapy is not

feasible

-Brachytherapy only at specialized centers

or in setting of

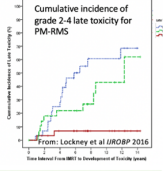

Late Effects

- Cosmetic defects

(ex. Facial

hypoplasia)

-Vision/hearing

-Dental

complications

-Second cancers

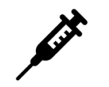

Surgery

-Limited to biopsy or

relapsed setting 